\title{
Present View of the Management and Tasks in the Celiac Disease Field: From Diagnosis to Therapy
}

\author{
Peter Makovicky ${ }^{1, *}$, Gabriel Samasca ${ }^{2}$ \\ ${ }^{1}$ Czech University of Life Sciences in Prague, Department of Veterinary Sciences, Prague, Czech Republic \\ ${ }^{2}$ Iuliu Hatieganu University of Medicine and Pharmacy, Institution of Immunology, Cluj-Napoca, Romania \\ *Corresponding author: pmakovicky@email.cz
}

Received November 17, 2013; Revised November 23, 2013; Accepted November 27, 2013

\begin{abstract}
Celiac disease is a disease where both children and adults are affected. It symptoms were previously described in Galen times. Today we recognize its starter, pathological mechanism, and also its atypical behaviour. There is only one causal therapeutic model in terms of compliance with the principles of lifelong strict gluten-free diet. It is an under-diagnosed disease. Further steps should be associated with the improvement of diagnosis and detection of all global forms of disease followed by proper care for patients, including prevention. The second part of the task is to support basic and applicable research with outcomes for further practice and therapeutic outcomes. It is an interdisciplinary issue with lack of its own publishing space. Given these facts, collaboration with the broader but highly professional Editorial Board found a new journal was: International Journal of Celiac Disease, which aims to address the issue at a high professional level, so the results could be applied in practice and science as well.
\end{abstract}

Keywords: celiac disease, diagnostics, research

Cite This Article: Peter Makovicky, and Gabriel Samasca, "Present View of the Management and Tasks in the Celiac Disease Field: From Diagnosis to Therapy.” International Journal of Celiac Disease 1, no. 1 (2013): 35. doi: 10.12691/ijcd-1-1-2.

\section{Introduction}

Celiac disease (CD) is a disease where both children and adults are affected. In modern world, it is a wellknown disease that is characterized by intolerance to gluten. Even during my studies, school taught us that the disease is rare, specific to the pediatric population and that over the time of it all the children will "surely grow" from it. This theory was based on assumption that this allergic food intolerance is temporary. Further studies revealed that its incidence among the European population is much higher than originally expected. Results of the first demographic study reported the incidence of Italy (1:250), southwest Ireland (1:300), Scotland (1:850), Sweden (1:960), the former Czechoslovakia (1:1000) and North America (1:5000) and that in Africa and Asia, the disease is less common. Having said, the time has shown that some countries in Europe are in detecting pediatric, or adult $\mathrm{CD}$, ahead than the others. Most likely it's because of outputs of the leading scientists in laboratories, currently still leading the teams that discovered that the disease may contain its so-called atypical symptomatology, where the typical form is just the tip of the iceberg $[5,6]$. Prerequisites of perfectly written article from the pen of Professor Catassi et al. [2] on the detection of new cases began to be more and more realistic. Nowadays, we know that it is a common disorder and the differences in incidence are both the result of the level of diagnostics where outcomes of the disease are neglected. Expansion of screening utilities and more sensitive methods to other European markets as well as other countries, states, continents causes increase of $\mathrm{CD}$ on a global scale. Currently we know estimation that: one hundred people equal one affected individual (1:100). However, some countries still report significantly lower prevalence than neigh boring countries and its estimate. This means that the disease is under-diagnosed. Given these facts, professional independent companies adopted the conclusions of which implies the need of a higher level of diagnostics, further intensification of education among the professionals and general public, and even greater need for support of the study and disease outcomes, which would lead to practical knowledge. It is known that the only causal therapy is lifelong strict compliance with the principles of gluten-free diet. There are no compromises while being on this diet. This brief introduction above shows that currently there are more priority issues among the professionals and the general public, hand by hand with other problems that are affecting our lives every day, overshadowing those with $\mathrm{CD}$. In this article, we would therefore like to present you our personal opinions about present view of the management and tasks in the $C D$ field.

\section{Interdisciplinary of the Problem}

"From diagnosis to therapy" is the main slogan of our editorial, in which we would like to define the main tasks in the field of CD. Time has passed, from the era of the 
discovery of the immunological basis of disease. New facts were revealed and pathology of the disease became known. Unfortunately, in our reality, there are still more questions to ask, than answers to give. Today we know that it is an interdisciplinary problem that is no longer the prerogative of pediatric gastroenterology. However, in our opinion we believe that problematic has much more complex profile of diagnostic, clinical, eventually research portfolio. Therefore, the discussions are very common among breeders, plant geneticists, primary agricultural producers, furthermore producers and food [3]. To minimize the toxicity of the grain that is the starter, it was looking for ways to completely remove the parts of the grain, characterized by its toxicity toward patients. It has been proven that the pathological response occurs even with residual amounts of gluten. In this context, daily intake of gluten for patients is still debatable in the field of which is already considered to be harmless and which pathological. Differences within the world and even intraEuropean quotas only bring chaos into this problem. Marking of gluten-free products also doesn't bring anything easy toward customers. A general method for labelling of gluten-free food on the pack aging that the product is gluten-free, should not contain any gluten, yet a few lines further the customer learns that it actually contains gluten. As for a biological research, it is undisputed that the most important benefits originate from immunological laboratories that support molecular biology and pathology completely changed the view of the pathophysiology of the disease $[1,4,8,10,13,14]$. Participation of leading geneticists' research resulted in the conclusions, which are useful in the diagnosis and revealed a percentage of predispositions in the population. Relationships were found among oncological diseases, as well as relationships between different clinical units and CD. Neurobiological basis of the relationship between mental health problems and celiac disease, celiac disease and aging had been revealed. Information listed above is just selected ones, well known for those who are generally involved in this problem. All of these and other outputs, either directly or indirectly, signed the repeatedly revised and published guidelines ESPGHAN, which should be for the diagnosis of celiac disease globally mandatory [7]. The ever-increasing percentage of diagnosed patients with their demands to quality of life provoked an economic discussion. Questions considering compensation claims for special foods insurers, and possibly other compensation ultimately are questions around the promotion of independent central clubs with priority of promotion and informational character. It is well known that these aspects show greater differences both between continents and within European countries.

\section{Tasks and Four-step Individual Management}

Within our short text, currently in the field of CD, there are two strategic tasks. The first involves an exact diagnosis and subsequent concern with the implementation of the level worldwide level and the second lies in the study of the disease with applicable and therapeutic outcomes for practice. Today, there are several versions of the model of how to diagnose the disease, within the typology of groups at risk. Literature shows different proposals toward this disease. Some of them prefer the wider application of serological screening, based on the results of previous greater studies [12]. Discussions are still held among the choice of forms of screening with the best combination, including good specificity, sensitivity and lower cost of a shorter period of time from receipt of materials, through processing to delivery of the actual outcome. Elsewhere, there is a support for the implementation of some special genetic test [11]. Other authors recommend that each endoscopy biopsy sampling implement should contain several samples of small intestine with special needs in functional dyspepsia, or even regardless of the cause of examination $[9,15]$. It seems that surface form of screening is not the way, but rather to identify ways to diagnose atypical forms. One of the problems, which fails to recognize the signs of atypical forms of CD, possibly even longer under several annual search for the right diagnosis lies in the fact that there is no applicable model, which would be useful for specialists of various approvals.

In our opinion, patients should be classified into groups and addressed to different groups of clinicians. Application would also help us to diagnose algorithm and detect hidden forms, including good care of diagnosed patients, including the prevention of further complications. According to us, the individual management of CD should include four groups of patients, which the clinician may encounter.

The first involves undiagnosed with the distribution of the pediatric group and adult group with instruments for the resolution of CD-like enteropathy. From the perspective of diagnosis identified this group should be addressed as risky and addressed to different professionals, comprising doctors of first contact through various specialists.

The second group includes newly-diagnosed patients with the classification of the pediatric population and late diagnosed adult group.

The third group includes patients already diagnosed with CD with the division of good clinical response to a gluten-free diet, further more without a relevant response to a gluten-free diet.

The fourth group consists patients with other associated diseases, that can be treated with either CD or that require specific, individual, or a different therapy. This classification seems to us to be clear, where each group requires a different approach, which may lead to a result of several experts of different approvals and therapeutic plan, which should be identify by a specialist, gastroenterologist.

As for the second so-called therapeutic tasks, it should be a part of the controlled research. Further research should take an intense path through a wider cooperation. Results should contribute to a better understanding of patho-physiological mechanisms, genetic predisposition and the interpretation of the relationships between different clinical units and eventually to alternative therapeutic procedures, possibly to other forms of vital therapy. Were commend to support all forms of applicable research output sin clinical practice, further theoretical basic research, through applicable research trends to achieve outcomes that could be helpful with those effected with CD. 


\section{Conclusion}

From our study, CD is an interdisciplinary problem. We believe that we deserve our own journal. This need exists here for a long period of time and those, who are interested in this marvellous problem, know it. Therefore, we decided to co-participate in this task. From the very beginning we had this urge of trying to come out with the greater good for everyone interested in this project. After a long discussion with wider knowledgeable experts, Editorin-Chief: Professor of Immunology, Gabriel Samasca the initiation of the establishment of a professional journal, where the first issue is available for of kind insight. In cooperation with widely profiled, but highly professional editorial board, we want to come out to greet all those who deal with the disease, but also to all who are waiting for the outcomes of these works. We therefore call upon all working groups and independent authors to contribute in this study, not to hesitate to share out puts of researchers from their own study, possibly, to contribute their own opinions, or their other interesting practical observations. Editorial Board welcomes and appreciates all the wellelaborated original works, recent reviews of the literature, short communications, letter to the editor, but also your critical comments, as well as announcements about book news, briefly treated news from the world of literature, reports on the activities of affiliated companies, information of professional congresses, and possibly information from technical descents. Every issue or problem that is primary concern is based on celiac disease, or is otherwise close to the load and the contents of the journal, is welcome. From this position, we would like to express the conviction that the journal will eventually have positive outcome by all the professionals, that it will become a source of valuable and interesting information as to the needs of clinical practice, various research institutions in order to fulfil our initial editorial content in the sense: from diagnosis to therapy.

\section{Statement of Competing Interests}

The authors have no competing interests. This article received no specific grant from any funding agency in the public, commercial and any other private profit sectors.

\section{Acknowledgement}

We acknowledge with thanks the help of Mrs. Alena Kralova for the English translation of this manuscript.

\section{References}

[1] Briani, C., Samaroo, D., Alaedini, A, Celiac disease: from gluten to autoimunity. Autoimmunity Reviews, 7 (8). 644-650. Sep.2008.

[2] Catassi, C., Ratsch, I.M., Fabiani, E., Rossini, M., Bordicchia, F.,Candela, F., Coppa, G.V., Giorgi, P.L, Coeliac disease in the year 2000: exploring the iceberg. Lancet, 343 (8891). 200-203. Jan.1994.

[3] Di Cagno, R., Barbato, M., Camillo, C., Rizzello, C.G., De Angelis, M., Giuliani, G., De Vincenzi, M., Gobbetti, M., Cucchiara, S, Gluten-free sourdough beat baked goods appeal safe for young celiac patiens: a pilot study. Journal of Pediatric Gastroenterology and Nutrition, 51 (6). 777-783. Dec.2010.

[4] Dieterich, W., Ehnis, T., Bauer, M., Donner, P., Volta, U., Riecken, E.O., Schuppan, D, Identification of tissue transglutaminase as the autoantigen of celiac disease. Natural Medicine, 3 (7). 797-801. 1997.

[5] Fasano, A., Catassi, C, Clinical practice. Celiac disease. New England Journal of Medicine, 367 (25). 2419-2426. Dec.2012.

[6] Green, P.H., Jabri, B, Coeliac disease. Lancet, 362 (9381). 383391. Aug.2003.

[7] Husby, S., Koletzko, S., Korponay-Szabo, I.R., Mearin, M.L., Phillips, A., Shamir, R., Troncone, R., Giersiepen, K., Branski, D., Catassi, C., Lelgeman, M., Maki, M., Ribes-Konincki, C., Ventura, A., Zimmer, K.P; ESPGHAN Working Group on Celiac Disease Diagnosis; ESPGHAN Gastroenterology Committee; European Society for Pediatric Gastroenterology, Hepatology, and Nutrition, European Society for Pediatric Gastroenterology, Hepatology, and Nutrition guidelines for the diagnosis of coeliac disease. Journal of Pediatric Gastroenterology and Nutrition, 54 (1). 136-160. Jan.2012.

[8] Chorzelski, T.P., Sulej, J., Tchorzewska, H., Jablonska, S., Beutner, E.H., Kumar, V, IgA class endomysium antibodies in dermatitis herpetiformis and coeliac disease. Annals of the New York Academy of Sciences, 420. 325-334. 1983.

[9] Kollarova, H., Pektor, R., Smajstra, V., Dvorackova, J., Shonova, O., Janoutova, G., Janout, V, Routine duodenal biopsy conducted in the course of gastroskopy - posibility of asymptomatic celiac disease detection. Czech-Slovac Gastroenterology and Hepatology, 61 (5). 245-248. Oct.2007.

[10] Marsh, M.N, Gluten, major histocompatibility complex, and the small intestine. A molecular and immunobiologic approach to the spectrum of gluten sensitivity ('celiac sprue'). Gastroenterology, 102 (1). 350-354.

[11] Mubarak, A., Spierings, E., Wolters, V., van Hoogstraten, I., Kneepkens, C.M., Houwen, R, Human leukocyte antigen DQ2.2 and celiac disease. Journal of Pediatric Gastroenterology and Nutrition, 56 (4). 428-430. Apr.2013.

[12] Mulder, C.J., Hadithi, M.M., Rostami, K., Goerres, M.S, Coeliac disease - has the time for routine mass screening? In 2002-20102020? Romanian Journal of Gastroenterology, 11 (3). 179-182. Sep.2002.

[13] Oberhuber, G., Granditsch, G., Vogelsang, H, The histopathology of coeliac disease: time for a standardized report scheme for pathologists. European Journal of Gastroenterology \& Hepatology, 11 (10). 1185-1194. Oct.1999.

[14] Prince, H.E, Evaluation of the INOVA diagnostics enzyme-linked immunosorbent Sasy kiks for measuring serum imunoglobulin $G$ (IgG) and IgA to deamidated gliadin peptides. Clinical and Vaccine Immunology, 13 (1). 150-151. Jan.2006.

[15] Soresi, M., Pirrone, G., Giannitrapani, L., Lacono, G., Di Prima, L., La Spada, E., Di Fede, G., Ambrosiano, G., Montalto, G., Carroccio, A, A key role for abdominal ultrasound examination in "difficult" diagnoses of celiac disease. Ultraschall in der Medizin, 32 (S1). S52-S61. Jan.2011. 